\title{
Interest of researchers in ultrasound systems for risk stratification of thyroid nodules (TIRADS): a systematic review
}

\author{
Pierpaolo Trimboli ${ }^{1,2} \oplus \cdot$ Giulia Ferrarazzo $^{3} \cdot$ Maurilio Deandrea $^{4} \cdot$ Chiara Camponovo $^{1} \cdot$ Francesco Romanelli $^{5}$. \\ Arnoldo Piccardo ${ }^{6} \cdot$ Cosimo Durante $^{7}$
}

Received: 8 September 2021 / Accepted: 2 November 2021 / Published online: 13 November 2021

(c) The Author(s) 2021

\begin{abstract}
Background A number of ultrasound risk stratification systems (RSSs) of thyroid nodule, often labeled as TIRADS (Thyroid Imaging Reporting and Data System), have been proposed. As a consequence, an increasing number of studies have been published on this topic. This systematic review was undertaken to answer specific questions in this field: how many articles and what type of studies have been published, which TIRADSs/RSSs have preferably been discussed, and what is the geographic distribution of the publications.

Methods The study was conducted according to PRISMA. A specific search algorithm was used. Defined selection criteria were applied.

Results 502 studies were finally included. The number of publications about TIRADSs/RSSs has increased over the time, being the Horvath TIRADS the most evaluated one. The first author of the article was from China in one fourth of cases.

Conclusions The number of scientific articles focused on TIRADSs/RSSs is high and it has been importantly increased over the time.
\end{abstract}

Keywords Thyroid nodule $\cdot$ Ultrasound $\cdot$ Thyroid Imaging Reporting and Data System (TIRADS) · Systematic review

Pierpaolo Trimboli and Giulia Ferrarazzo shared the co-authorship.

Pierpaolo Trimboli

pierpaolo.trimboli@eoc.ch

1 Servizio di Endocrinologia e Diabetologia, Ospedale Regionale di Lugano, Ente Ospedaliero Cantonale (EOC), Lugano, Switzerland

2 Facoltà di Scienze Biomediche, Università Della Svizzera Italiana (USI), Lugano, Switzerland

3 Nuclear Medicine, Ospedale Villa Scassi Hospital, Genoa, Italy

4 UO Endocrinologia, Diabetologia e Malattie del metabolismo, AO Ordine Mauriziano Torino, Torino, Italy

5 Department of Experimental Medicine, Sapienza University of Rome, Rome, Italy

6 Nuclear Medicine Department, Ente Ospedaliero “Ospedali Galliera", Genoa, Italy

7 Department of Translational and Precision Medicine, Sapienza University of Rome, Rome, Italy

\section{Introduction}

Ultrasound (US) is recognized as the most important modality for the assessment of malignancy risk of thyroid nodules. In fact, from the large experience of the last two decades, we know that thyroid nodules with specific US features, such as strong hypoechogenicity, taller-thanwide shape, irregular margins, microcalcifications, and extrathyroidal extension, are at high risk to be cancers $[1,2]$. On the contrary, those nodules without high-risk US features can be considered at low to intermediate risk and their likelihood to be a cancer significantly drops. Since 2009, a number of US risk stratification systems (RSSs) have been developed to classify thyroid nodule based on their sonographic patterns. The majority of these RSSs is labeled as TIRADS, as the acronym of Thyroid Imaging Reporting and Data System, in agreement to the case of the breast US (i.e., BIRADS). The first proposal came from a Chilean group [3]; afterwards, later versions appeared in the main document of clinical practice guidelines for the management of thyroid nodules [4, 5] and in radiological position papers [6-9]. Table 1 summarizes 
Table 1 Major differences in terms of structure and high-risk characteristics among the RSSs considered in the present study

\begin{tabular}{llllll}
\hline & Chilean [3] & ATA [4, 5] & KTA [6, 7] & ETA [8] & ACR [9] \\
\hline $\begin{array}{l}\text { Structure } \\
\text { Number of classes }\end{array}$ & 6 & & & & \\
Pattern-/point-based & Pattern & Pattern & Pattern & Pattern & Point \\
High-risk features & & & & \\
Hypoechogenicity & Yes & Yes & Yes & Yes & Yes \\
Taller-than-wide & No & Yes & Yes & Yes & Yes \\
Irregular margins & Yes & Yes & Yes & Yes & Yes \\
Microcalcifications & Yes & Yes & Yes & Yes & Yes \\
Vascularization & Yes & No & No & No & No \\
Extra-thyroid extension & No & Yes & No & No & Yes \\
\hline
\end{tabular}

Yes/no means that the feature is or not considered in the RSS

ACR American College of Radiology, ATA American Thyroid Association, ETA European Thyroid Association, KTA Korean Thyroid Association the main differences among the RSSs. They include up to six categories, with the estimated risk of malignancy rising through the different classes. Four of these [the American, European and Korean Thyroid Association (ATA, ETA, KTA, respectively) and the Chilean RSSs] [3-8] were conceived based on the nodule's sonographic pattern, in that US features are combined into specific risk categories. The remaining one [American College of Radiology (ACR) RSS] [9] consists of summing points previously attributed to each US feature (a point-based scoring system). As for the high-risk features, only hypoechoic presentation, irregular margins and microcalcifications are included in all of them. With the advent of these systems, an increasing number of studies have been published on this topic and the majority of these attempted to compare the performance of these TIRADSs/RSSs. As the major finding, a meta-analysis reported that their reliability in selecting nodule for biopsy is quite similar [10]. In addition, the temporal stability of the initial risk assessed by TIRADSs/RSSs remains stable over the time [11].

The present study was aimed at systematically review the literature on this topic to account for the scientific production on TIRADSs/RSSs and ultimately address the following questions: how many articles and what type of studies have been published on the most diffused TIRADSs/RSSs [3-9]; which TIRADSs/RSSs have preferably been compared and discussed in the published meta-analyses, original articles and other kind of studies; what is the geographic distribution of the publications in this field.

\section{Materials and methods}

\section{Review conduction}

The systematic review was conducted according to the PRISMA statement [12]. The checklist is reported as supplemental file.

\section{Search strategy}

A three-step search strategy was planned. First, keywords were identified in PubMed. Second, PubMed and Scopus were searched using the following algorithm "ATA ultrasound system OR ("TIRADS" OR "TI-RADS") OR ("EU-TIRADS" OR "EU-TI-RADS") OR ("K-TIRADS" OR "K-TI-RADS") OR ("ACR-TIRADS" OR "ACR-TI-RADS")". Third, any citation of the above RSSs was recorded. The last search was performed on July 10th, 2021. No language neither time restriction was adopted. Two investigators (GF, PT) independently searched papers, screened titles and abstracts of the retrieved records, and finally selected the included references.

\section{Data extraction}

The following information was extracted independently and in duplicate by two investigators (GF, PT) in a piloted form: first author, year of publication, country of the first author, study type, RSS(s) under investigation among those were considered [3-9]. Data were cross-checked and discrepancies were mutually discussed. 


\section{Data analysis}

The number of articles evaluating each TIRADS/RSS was calculated. Then, the overall number of articles evaluating all systems was obtained. The kind or articles (i.e., original paper, meta-analysis, etc.) was recorded.

\section{Results}

\section{Records found}

According to above search algorithm, a number of 538 records were found and, after exclusion of duplicates and articles not meeting the inclusion criteria or not in the area of interest, 502 studies were finally included in the systematic review.

\section{Article type}

Among the articles included in the present systematic review, 13 were systematic review with meta-analysis, 424 were original paper, 34 were narrative review, 4 were guidelines or practical recommendations, and 27 were other kind of article (i.e., 12 comment, 2 editorial article, 13 case report).

\section{Analysis of inclusion of the different TIRADSs/RSSs in the published studies}

The number of articles evaluating at least one RSS was increasing over the time, with a sharp steepening of the curve starting from 2017. This trend applies to all types of RSSs. Overall, the TIRADSs/RSSs [3-9] were evaluated as follows: Horvath TIRADS 213 times, ACR-TIRADS 190, ATA 144, K-TIRADS 99, and EU-TIRADS 71. Two articles included all the five systems. Also, there were 21 articles evaluating four systems (i.e., ATA, ACR-, K, and EU-TIRADS), 26 articles three systems, 92 articles two systems, and 361 evaluating only one of them. Figure 1 illustrates the number of articles published up to 2020, while data of 2021 were not displayed because referring to the first half of the year only.

As showed in Fig. 2, the trend analysis of cumulative number of articles evaluating any system showed a progressive increase over the time for all TIRADSs/RSSs; as above mentioned, since data of 2021 were referred to the first half of the year, they were not included.

\section{Geographic distribution}

The first author of the 502 articles was from 45 different countries. He was from China in $25.7 \%$, USA in $15.9 \%$, Republic of Korea in $14.3 \%$, Italy in $6.9 \%$, and from other 41 countries in less than $5 \%$ of cases. Considering the Continents, the first author was Asian in 210 cases, European in 138, American in 113, Middle Eastern in 24, African in 11, and Oceanian in 6. Figure 3 illustrates the filled map of these findings.

\section{Discussion}

The present study was conceived to analyze the interest of researchers in the US systems for the risk stratification of the thyroid nodules. With this objective, we systematically searched for the published articles evaluating the first system proposed by Horvath et al. [3], the system included in ATA guidelines for management of nodule and cancer $[4,5]$, and the most recognized TIRADS, such K- [6, 7], EU- [8], and ACR-TIRADS [9]. The results of the present systematic review may have the chance of arouse interest in researchers

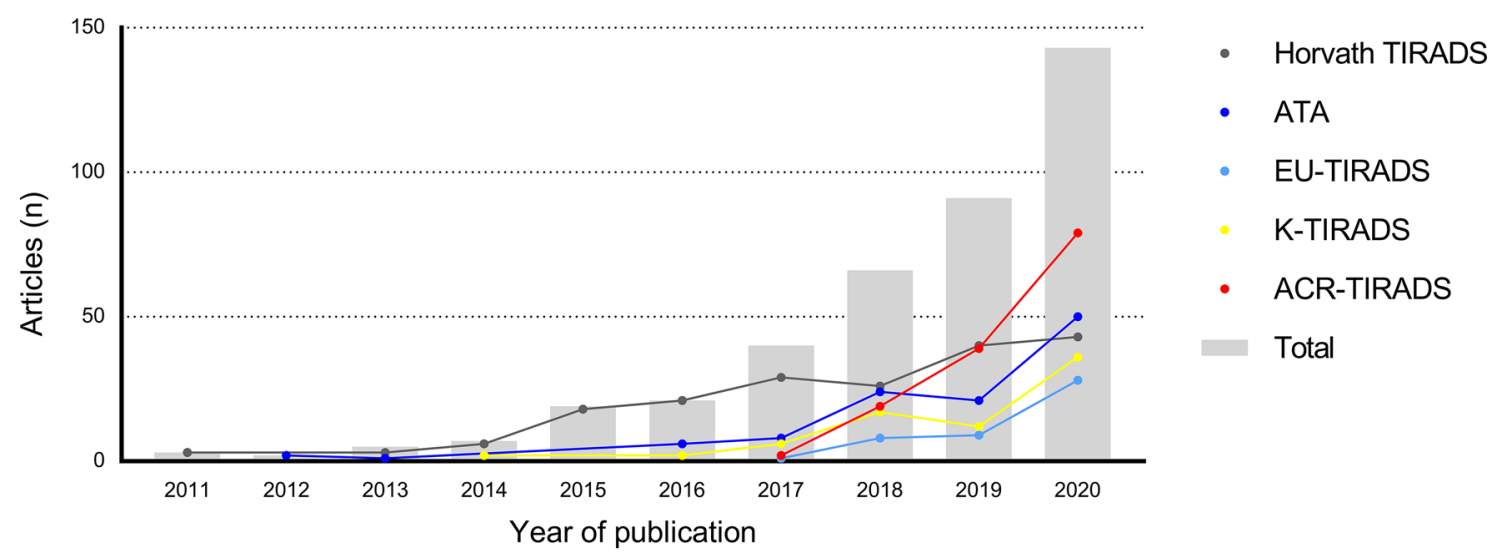

Fig. 1 Number of articles published evaluating the five herein considered TIRADSs/RSSs [3-9]. Each point of each curve represents the occurrences of any TIRADS/RSS in the articles published in that year 


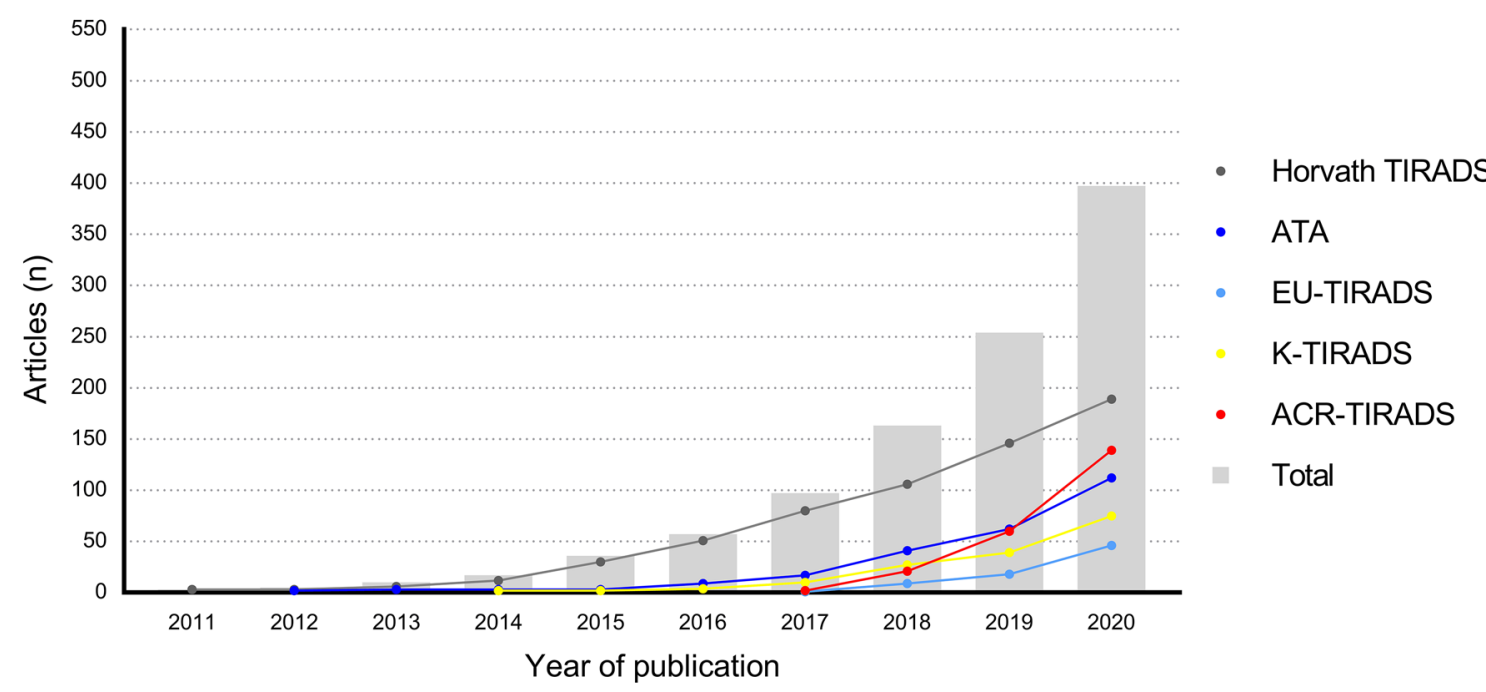

Fig. 2 Cumulative number of articles over the time evaluating the TIRADSs/RSSs. Each point of each curve represents the total number of articles evaluating any TIRADS/RSS at that moment

Fig. 3 Filled map of the world prevalence of studies focused on TIRADSs/RSSs found by present systematic review. The number of published articles per country was the following: China 129, USA 80, Republic of Korea 72, Italy 35, Germany 18 , France 16 , India 15 , Turkey 15, Brazil 15, Switzerland 13, Poland 12, Canada 10, Australia 4, Egypt 4, UK 4, Israel 4, Spain 4, Philippines 3, Singapore 3, Taiwan 3, Norway 3, Romania 3, Russia 3, South Africa 3, Iran 3, Mexico 3, Argentina 2, Colombia 2, New Zealand 2, Saudi Arabia 2, Thailand 2, Malaysia 1, Pakistan 1, Austria 1, Belgium 1, Bulgaria 1, Czech Republic 1, Hungary 1, Ireland 1, Portugal 1, Cameroon 1, Chile 1, Nigeria 1, Tunisia 1, Uganda 1, Denmark 1

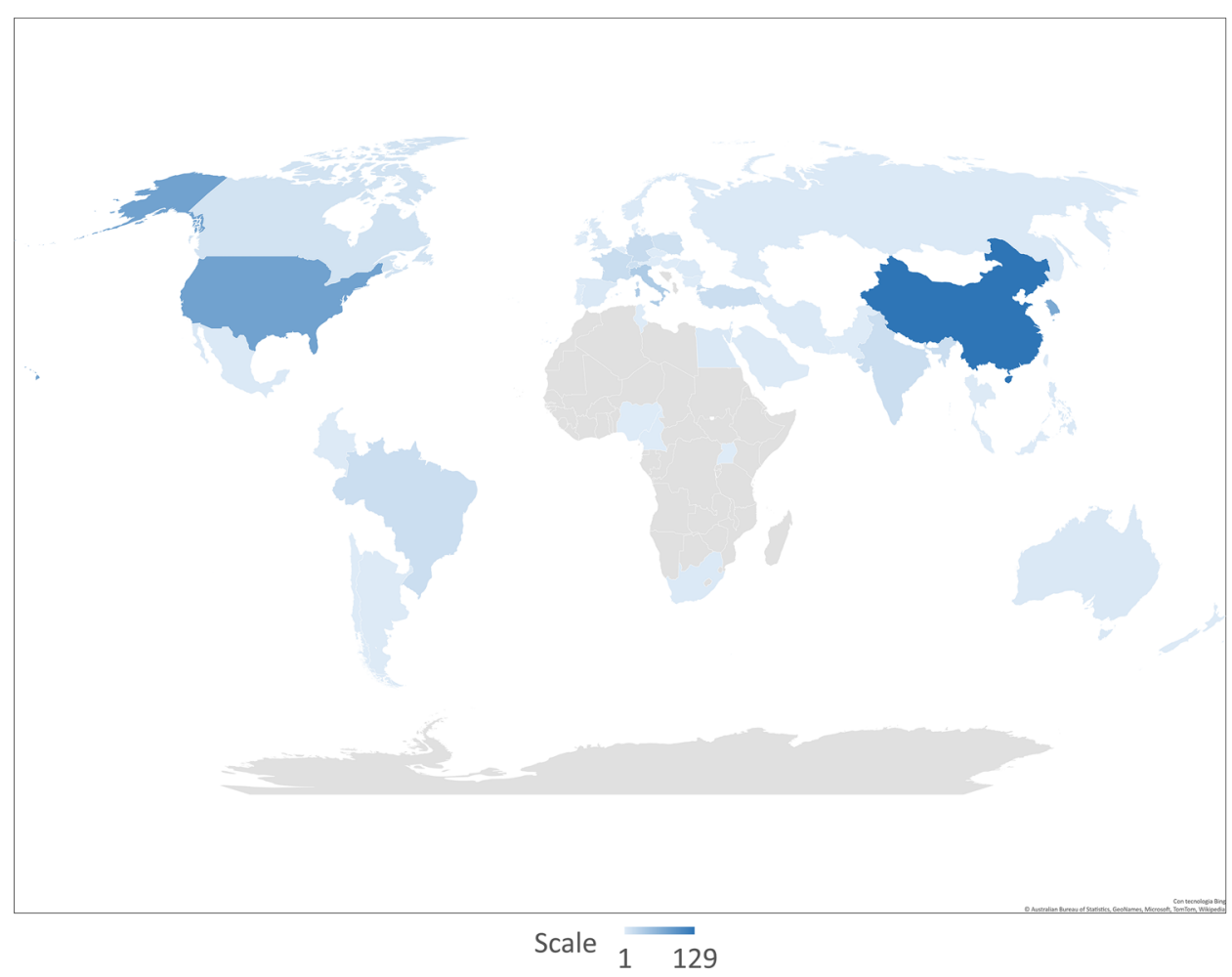

and readers focused on this field, and in the panelists of the systems themselves.

Three main findings were reported and are worthy of attention. First, a large number of articles were found since the primordial RSS released in 2009 [3]. They account for over 500 studies, the majority being original papers, but with a not negligible number $(n=13)$ of meta-analyses. Second, a fast-growing number of studies evaluating or discussing the TIRADSs/RSSs was observed over the time. This trend is particularly relevant for ACR-TIRADS [9], despite its more recent appearance in paper databases. It is noteworthy the continuous interest for the Horvath TIRADS, which has now been proposed over 10 years ago and, unlike the other systems, has not been proposed or endorsed by a scientific society. Third, the body of publications is distributed across Western and Eastern countries. Notably, investigators are 
Asian in $41 \%$ of cases and, in particular, from China in one fourth. As for the latter, in 2020 a Chinese RSS has been released (i.e., C-TIRADS) [13]: the interest of researchers in this system will become clearer in the next future. Overall, this remarkable scientific activity that revolves around RSSs suggests the interest and the need of the scientific community in testing and/or validating tools that allow to standardize and, hopefully, facilitate the management of thyroid nodules. This is undoubtedly facilitated by the epidemic numbers that thyroid nodular disease is assuming worldwide [14] and the move towards an individualized approach tailored on the patients' risk [15].

Unfortunately, the present study cannot answer some other questions arising out clinical practice. In fact, whether these systems are used in routine practice is not known. Furthermore, whether these systems are easy-to-use during clinical assessment of patients and if one system is easier and more handle than the other ones is an unexplored matter. In this context, we should consider the heterogenous clinical scenarios across different continents and countries. For instance, in United States and other countries neck US is generally performed by technicians or nurses and, within this context, using a schematic point-based RSS, such as ACR-TIRADS, can help the correct acquisition of images and the following interpretation.

Some potential limitations of the present data should be addressed. First, we created a specific algorithm (see above) and we found a large number of articles. The fact that a 7\% (36/538) of records initially found was excluded because it did not meet the inclusion criteria should represent an indirect proof that our data can be considered as reliable. However, the present was not a pure librarian search, since it was undertaken with the perspective to give the users of TIRADSs/RSSs an almost complete vision about their diffusion in the scientific research databases. Second, the count per country of published articles was performed considering the affiliation of the first author. In addition, series with potential to overlap between two or more articles were not considered. This means that some particular cases (i.e., multicenter series) might be neglected. Third, here we considered only the most diffused TIRADSs/RSSs [3-9]. Even if this choice was arbitrary, we feel that this was in agreement with the worldwide diffusion of the TIRADSs/RSSs in clinical practice. Fourth, two systems that we included in our review were revised over the time and we considered both versions of them [4-7]. This might slightly affect the data of these RSSs.

\section{Conclusions}

The present systematic review showed that the number of scientific articles focused on US systems for risk stratification of thyroid nodules is high and has been importantly increased over the time. In addition, the interest of researchers in this field is well balanced between Western and Eastern countries. These data reveal the worldwide interest of researchers in testing and improving the accuracy of RSSs, and vindicate the need for a collective and systematic effort to work out a universal classification system. A task force of experts representing all scientific societies working in the field is currently engaged in efforts to develop a unified, evidence-based, international system (i.e., I-TIRADS) [16].

Author contributions PT conceived the study. All authors contributed to the development of the selection criteria, the risk of bias assessment strategy and data extraction criteria. GF and PT developed the search strategy, performed database search, acquired the data, analyzed the data and drafted the manuscript. PT and FR provided statistical expertise. CD performed a critical review. All Authors read, provided feedback, and approved the final manuscript.

Funding Open access funding provided by Università della Svizzera italiana. This research received no external funding.

\section{Declarations}

Conflict of interest The authors declare that they have no conflict of interest.

Informed consent For this type of study, informed consent is not required.

Research involved with human or animal participants This article does not contain any studies with human or animal subjects performed by the any of the authors.

Open Access This article is licensed under a Creative Commons Attribution 4.0 International License, which permits use, sharing, adaptation, distribution and reproduction in any medium or format, as long as you give appropriate credit to the original author(s) and the source, provide a link to the Creative Commons licence, and indicate if changes were made. The images or other third party material in this article are included in the article's Creative Commons licence, unless indicated otherwise in a credit line to the material. If material is not included in the article's Creative Commons licence and your intended use is not permitted by statutory regulation or exceeds the permitted use, you will need to obtain permission directly from the copyright holder. To view a copy of this licence, visit http://creativecommons.org/licenses/by/4.0/.

\section{References}

1. Durante C, Grani G, Lamartina L, Filetti S, Mandel SJ, Cooper DS (2018) The diagnosis and management of thyroid nod-ules: a review. JAMA 319:914-924. https://doi.org/10.1001/jama.2018. 0898

2. Trimboli P (2021) Ultrasound: the extension of our hands to improve the management of thyroid patients. Cancers (Basel) 13:567. https://doi.org/10.3390/cancers 13030567

3. Horvath E, Majlis S, Rossi R, Franco C, Niedmann JP, Castro A, Dominguez M (2009) An ultrasonogram reporting system for thyroid nodules stratifying cancer risk for clinical management. 
J Clin Endocrinol Metab 94:1748-1751. https://doi.org/10.1210/ jc. 2008-1724

4. Cooper DS, Doherty GM, Haugen BR, Kloos RT, Lee SL, Mandel SJ, Mazzaferri EL, McIver B, Pacini F, Schlumberger M, Sherman SI, Steward DL, Tuttle RM (2009) Revised American thyroid association management guidelines for patients with thyroid nodules and differentiated thyroid cancer. Thyroid 19:1167-1214. https://doi.org/10.1089/thy.2009.0110

5. Haugen BR, Alexander EK, Bible KC, Doherty GM, Mandel SJ, Nikiforov YE, Pacini F, Randolph GW, Sawka AM, Schlumberger M, Schuff KG, Sherman SI, Sosa JA, Steward DL, Tuttle RM, Wartofsky L (2016) 2015 American thyroid association management guidelines for adult patients with thyroid nodules and differentiated thyroid cancer: the American thyroid association guidelines task force on thyroid nodules and differentiated thyroid cancer. Thyroid 26:1-133

6. Moon WJ, Baek JH, Jung SL, Kim DW, Kim EK, Kim JY, Kwak JY, Lee JH, Lee JH, Lee YH, Na DG, Park JS, Park SW (2011) Korean society of thyroid radiology (KSThR); Korean society of radiology. Ultrasonography and the ultrasound-based management of thyroid nodules: consensus statement and recommendations. Korean J Radiol 12:1-14. https://doi.org/10.3348/kjr.2011.12.1.1

7. Shin JH, Baek JH, Chung J, Ha EJ, Kim JH, Lee YH, Lim HK, Moon WJ, Na DG, Park JS, Choi YJ, Hahn SY, Jeon SJ, Jung SL, Kim DW, Kim EK, Kwak JY, Lee CY, Lee HJ, Lee JH, Lee JH, Lee KH, Park SW, Sung JY (2016) Korean society of thyroid radiology (KSThR) and Korean society of radiology. Ultrasonography diagnosis and imaging-based management of thyroid nodules: revised Korean society of thyroid radiology consensus statement and recommendations. Korean J Radiol 17:370-395. https://doi. org/10.3348/kjr.2016.17.3.370

8. Russ G, Bonnema SJ, Erdogan MF, Durante C, Ngu R, Leenhardt L (2017) European thyroid association guidelines for ultrasound malignancy risk stratification of thyroid nodules in adults: the EU-TIRADS. Eur Thyroid J 6:225-237

9. Tessler FN, Middleton WD, Grant EG, Hoang JK, Berland LL, Teefey SA, Cronan JJ, Beland MD, Desser TS, Frates MC, Hammers LW, Hamper UM, Langer JE, Reading CC, Scoutt LM, Stavros AT (2017) ACR thyroid imaging, reporting and data system (TI-RADS): white paper of the ACR TI-RADS committee. J Am Coll Radiol 14:587-595

10. Castellana M, Castellana C, Treglia G, Giorgino F, Giovanella L, Russ G, Trimboli P (2020) Performance of five ultra-sound risk stratification systems in selecting thyroid nodules for FNA. J Clin Endocrinol Metab 105(5):dgz170. https://doi.org/10.1210/clinem/ dgz170 (PMID: 31690937)

11. Grani G, Lamartina L, Biffoni M, Giacomelli L, Maranghi M, Falcone R, Ramundo V, Cantisani V, Filetti S, Durante C (2018) Sonographically estimated risks of malignancy for thyroid nodules computed with five standard classification systems: changes over time and their relation to malignancy. Thyroid 28:1190-1197. https://doi.org/10.1089/thy.2018.0178

12. Page MJ, McKenzie JE, Bossuyt PM, Boutron I, Hoffmann TC, Mulrow CD, Shamseer L, Tetzlaff JM, Akl EA, Brennan SE, Chou R, Glanville J, Grimshaw JM, Hróbjartsson A, Lalu MM, Li T, Loder EW, Mayo-Wilson E, McDonald S, McGuinness LA, Stewart LA, Thomas J, Tricco AC, Welch VA, Whiting P, Moher D (2021) The PRISMA 2020 statement: an updated guideline for reporting systematic reviews. BMJ 372:n71. https://doi.org/10. 1136/bmj.n71

13. Zhou J.; Yin L.; Wei X.; Zhang S.; Song Y.; Luo B.; Li J.; Qian L.; Cui L.; Chen W.; Wen C.; Peng Y.; Chen Q.; Lu M.; Chen M.; Wu R.; Zhou W.; Xue E.; Li Y.; Yang L.; Mi C.; Zhang R.; Wu G.; Du G.; Huang D.; Zhan W. Superficial Organ and Vascular Ultrasound Group of the Society of Ultrasound in Medicine of the Chinese Medical Association; Chinese Artificial Intelligence Alliance for Thyroid and Breast Ultrasound (2020) Chinese guidelines for ultrasound malignancy risk stratification of thyroid nodules: the C-TIRADS. Endocrine 2020(70):256-279. https://doi.org/10. 1007/s12020-020-02441-y

14. Li M, Dal Maso L, Vaccarella S (2020) Global trends in thyroid cancer incidence and the impact of overdiagnosis. Lancet Diabetes Endocrinol 8:468-470. https://doi.org/10.1016/S2213-8587(20) 30115-7

15. Grani G, Sponziello M, Pecce V, Ramundo V, Durante C (2020) Contemporary thyroid nodule evaluation and management. J Clin Endocrinol Metab 105:2869-2883. https://doi.org/10.1210/ clinem/dgaa322

16. 2019 ATA Annual Meeting. Short call poster 76 (2019) https:// doi.org/10.1089/thy.2019.29087.sc.abstracts

Publisher's Note Springer Nature remains neutral with regard to jurisdictional claims in published maps and institutional affiliations. 